\title{
Spatial pattern analysis of nuclear migration in remodelled muscles during Drosophila metamorphosis
}

\author{
Kuleesha $a^{1,2^{*}}$ (D) Lin Feng ${ }^{1}$ and Martin Wasser ${ }^{2,3}$
}

\begin{abstract}
Background: Many human muscle wasting diseases are associated with abnormal nuclear localization. During metamorphosis in Drosophila melanogaster, multi-nucleated larval dorsal abdominal muscles either undergo cell death or are remodeled to temporary adult muscles. Muscle remodeling is associated with anti-polar nuclear migration and atrophy during early pupation followed by polar migration and muscle growth during late pupation. Muscle remodeling is a useful model to study genes involved in myonuclear migration. Previously, we showed that loss of Cathepsin-L inhibited anti-polar movements, while knockdown of autophagy-related genes affected nuclear positioning along the medial axis in late metamorphosis.

Results: To compare the phenotypic effects of gene perturbations on nuclear migration more objectively, we developed new descriptors of myonuclear distribution. To obtain nuclear pattern features, we designed an algorithm to detect and track nuclear regions inside live muscles. Nuclear tracks were used to distinguish between fast moving nuclei associated with fragments of dead muscles (sarcolytes) and slow-moving nuclei inside remodelled muscles. Nuclear spatial pattern features, such as longitudinal (lonNS) and lateral nuclear spread (latNS), allowed us to compare nuclear migration during muscle remodelling in different genetic backgrounds. Anti-polar migration leads to a lonNS decrease. As expected, lack of myonuclear migration caused by the loss of $C p 1$ was correlated with a significantly lower IonNS decrease. Unexpectedly, the decrease in IonNS was significantly enhanced by Atg9, Atg5 and Atg18 silencing, indicating that the loss of autophagy promotes the migration and clustering of nuclei. Loss of autophagy also caused a scattering of nuclei along the lateral axis, leading to a two-row as opposed to single row distribution in control muscles. Increased latNS resulting from knockdown of Atg9 and Atg18 was correlated with increased muscle diameter, suggesting that the wider muscle fibre promotes lateral displacement of nuclei from the medial axis during polar migration.

Conclusions: We developed new nuclear features to characterize the dynamics of nuclear distribution in time-lapse images of Drosophila metamorphosis. Image quantification improved our understanding of phenotypic abnormalities in nuclear distribution resulting from gene perturbations. Therefore, in vivo imaging and quantitative image analysis of Drosophila metamorphosis promise to provide novel insights into the relationship between muscle wasting and myonuclear positioning.
\end{abstract}

Keywords: Drosophila metamorphosis, Myonuclear localization, Nuclear segmentation, Nuclear tracking, Classification, Feature generation

\footnotetext{
* Correspondence: kuleesha26@gmail.com

${ }^{1}$ School of Computer Science and Engineering, Nanyang Technological

University, Singapore, Singapore

${ }^{2}$ Imaging Informatics Division, Bioinformatics Institute (BII), Agency for

Science, Technology and Research ( $A^{*}$ STAR), Singapore, Singapore

Full list of author information is available at the end of the article
} 


\section{Background}

Skeletal muscle fibres are large multinucleated cells. $\mathrm{Nu}$ clei inside of muscle cells (myonuclei) are thought to be positioned such that the local nuclear-cytoplasmic ratio remains constant [1]. One reason for this behaviour is that a nucleus can only support a fixed volume of cytoplasm, called a myonuclear domain (MND) [2], due to the limited distance that proteins can be transported inside cells [3]. Therefore, in healthy muscles, nuclei are expected to be evenly distributed. A quantitative study on the spatial distribution of nuclei in mice has confirmed that the myonuclei are not randomly distributed and are arranged in a row-like formation, indicating that the nuclei could be repelling each other to minimize the transport distance [4]. Unlike healthy muscles, several studies have revealed abnormal MND sizes in hypertrophic and atrophied muscles [5, 6]. Centrally positioned nuclei have been observed in many muscle disorders, including central nuclear myopathies [7] and muscular dystrophy $[8,9]$. Previous studies have shown that nuclear envelope proteins play a role in regulating nuclei positioning [10, 11]. In a study on Drosophila larvae, the KASH mutants showed impaired locomotion and aggregation of the myonuclei [12]. Loss of JNK signalling also caused clustering of nuclei and large regions in muscles devoid of nuclei [13]. Despite numerous studies on nuclear positioning, its role in muscle function remains unclear.
Previously, we reported that, during Drosophila metamorphosis, which last 4 to 5 days, the nuclei in abdominal dorsal internal oblique muscles (DIOM), also referred to as persistent muscles show changes in myonuclear distribution [14]. In larval and prepupal stages, nuclei show an even distribution within the muscle fibres (Fig. 1). After head eversion (HE), taking place approximately $12 \mathrm{~h}$ after puparium formation, most skeletal muscles undergo programmed cell death and become fragmented, while persistent muscles survive into adulthood. We will refer to nuclei inside the persistent muscles as internal nuclei and nuclei inside sarcolytes (muscle fragments) as external nuclei. In the first 2 days of pupation after HE, persistent muscles undergo atrophy and their nuclei start migrating in an anti-polar fashion towards the centre of the muscle. At mid-pupation, the direction of myonuclear migration reverses and the nuclei move back to the poles while positioning themselves along the medial axis of muscles. While the muscle diameter increases in late pupation, the nuclei remain anchored in a single-row formation along the medial axis. Myonuclear migration was also reported to occur in early myogenesis when mouse myoblasts fuse with myotubes [15] and Drosophila embryonic myoblasts fuse with founder cells [16], suggesting that muscle remodelling could be interpreted as dedifferentiation of mature muscles into a myotube-like state. In a pilot forward genetics RNAi screen, we also identified the first genes

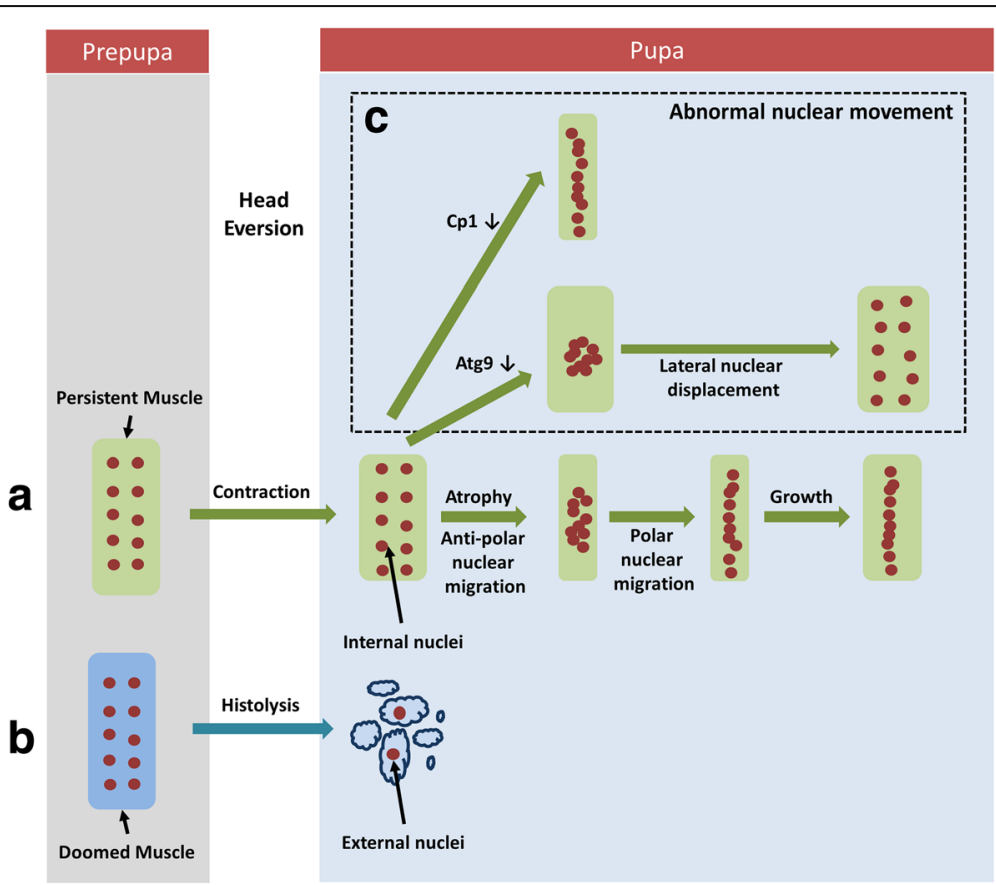

Fig. 1 Schematic diagram explaining different stages of nuclear localization. a In persistent muscles, the nuclear positioning transitions from an initial two-row like formation in prepupae to a clustered distribution in mid-pupation, and lastly a one row formation in late pupation. b A sub-set of $\mathrm{DIOM}$ undergo histolysis and create muscle debris with nuclei inside them. These nuclei form the external nuclei. c Knockdown of Cp1 and Atgs affect the myonuclear distribution 
that play roles in the migration and positioning of nuclei in remodelled muscles [14]. Silencing of $C p 1$, the gene encoding the homolog of the lysosomal proteases Cathpesin-L inhibited anti-polar migration in early migration. Knockdown of several autophagy-related genes (Atg5, Atg9, Atg12. Atg18) resulted in scattering of nuclei along the lateral axis in late metamorphosis, giving the appearance of a double-row formation (Fig. 1c, Additional file 1: Figures S1 \& S2) [14]. To better understand myonuclear distribution and compare the phenotypes resulting from genetic perturbations more objectively, new methods for the quantitative analysis of nuclear migration and localization are required. Spatial pattern analysis has been used to investigate the sub-cellular localization of centromeres [17], nuclei in multi-nucleated muscles [4], and nuclei in Drosophila embryos [18].

In this paper, we present our spatial pattern analysis algorithm to study the effects of genetic perturbations on the distribution of nuclei in remodelled Drosophila muscles during metamorphosis. Our method consists of two parts. First, we detect and track nuclei inside remodelled muscles expressing Mhc-tau-GFP and Histone-mKO to label cytoplasm and nuclei in two different colors. Since we analyse $2 \mathrm{D}$ projections of $3 \mathrm{D}$ image stacks, we need to classify nuclei inside muscle regions into slow-moving internal and fast-moving external nuclei. We demonstrate high accuracy for the segmentation, tracking and classification steps. Second, we calculate static and dynamic spatial pattern features of slow-moving nuclei corresponding to remodelled muscles. The longitudinal and lateral nuclear spreads and their changes over time helped us detect significant phenotypic variations between different genotypes that were not discernible by eyeballing. As such, quantitative analysis of nuclear migration and localization will improve the depth of phenotypic profiling in timelapse image analysis.

\section{Methods}

We used the UAS-GAL4 system to achieve targeted expression of fluorescent proteins and shRNA (small hairpin) in muscles. Muscle cytoplasm and nuclei were labelled using MHC-tau-GFP [19] and UAS-Histone 2Av-mKO [20], respectively. Mef2-GAL4 was used as a muscle specific driver [21]. All UAS-shRNA (small hairpin) transgenic lines were obtained from Transgenic RNAi Project (TRiP) collection [22]. In our experiment, we crossed female of reporter line MHC-tau-GFP/FM7-GFP; Mef2-GAL4, UAS-histone-mKO/TM6B Tb with male of UAS-GeneX$R N A i$ lines. We examined muscles of non-tubby progeny with genotype MHC-tau-GFP/+; Mef2-GAL4, UAS-histone$m K O / U A S-G e n e X-R N A i$. In our study, we used RNAi lines of the following genes: Chromator (Control, Bloomington Stock id: B-36084), Atg9 (B-34901), Atg18 (B-34714), Atg5
(B-34899), Atg12 (B-34675) and Cp1 (B-32932). The cross was done at $25^{\circ} \mathrm{C}$.

The protocol for sample preparation and microscopy has been previously described [23, 24]. Line scanning Zeiss LSM 5 Live microscope was used to perform live imaging of Drosophila pupae. 20-30 pupae were imaged simultaneously using multi location imaging feature of line scanning microscope. We performed imaging for a duration of 4-5 days. Images were collected at an interval of $30 \mathrm{~min}$. We also collected images of pupae at multiple focal planes. Two color channels were imaged: channel 1 with an excitation laser of $488 \mathrm{~nm}$, band path filter (BP) 500-525; and channel 2 with $532 \mathrm{~nm}$ laser line, BP 560675. The image acquisition was done with the following


$0.30 \mathrm{M} 27)$, pin hole size of $16.6 \mu \mathrm{m}$ and frame speed of 2 FPS. The images were of size $1024 \times 1024$ pixels, and the physical size of each pixel was $1.25 \times 1.25 \times 11.08 \mu \mathrm{m}$. The confocal imaging generated LSM files for each time point. LSM files of every time point of a pupae were concatenated into an ICS file using custom software [25]. For time series analysis of images, the 3D stacks of ICS files were converted into their $2 \mathrm{D}$ projections using the maximum intensity projection (MIP) method. The final result was a multi-tiff file in which each image represents a time point. This multi-tiff file was used as an input for nuclear spatial pattern analysis.

\section{Nuclear spatial pattern analysis pipeline}

A schematic diagram of the nuclear spatial pattern analysis has been shown in Fig. 2.

\section{Nuclear region extraction inside persistent muscles}

Apart from the nuclei inside the persistent muscles (internal nuclei), the nuclei inside dead muscle fragments (external nuclei) are also present in the pupa abdomen, as shown in Fig. 3a, a'. To calculate nuclear features, we require only the region occupied by internal nuclei. Therefore, after nuclear segmentation, removal of external nuclei from segmented nuclear regions is an important step in myonuclear spatial pattern analysis. The external nuclei which are located outside the persistent muscles can be removed easily using muscle boundary. However, it is difficult to remove external nuclei which appear to be inside persistent muscles due to $2 \mathrm{D}$ projection; while they are actually located above or below the persistent muscles. We could not use 3D images for removal of external nuclei due to low $\mathrm{z}$ resolution. To tackle these problems, we designed a new algorithm for extracting regions occupied by internal nuclei. Key techniques of the algorithm are:

1) Muscle Segmentation 


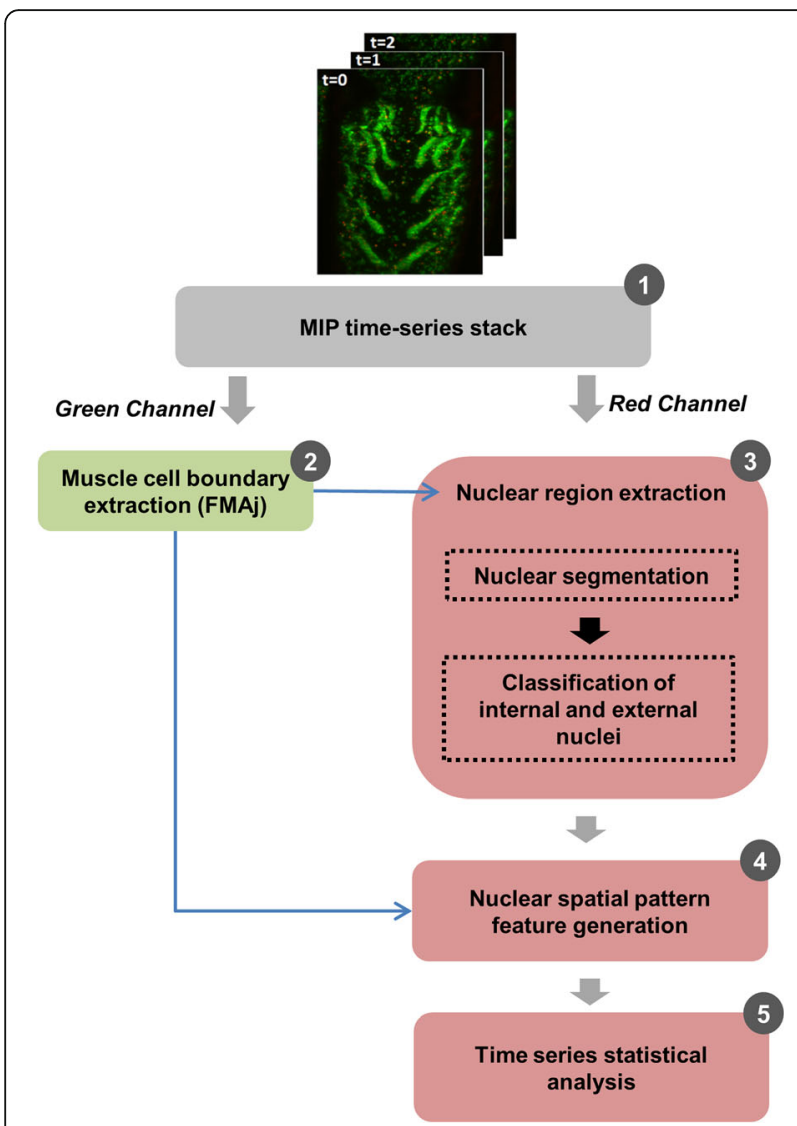

Fig. 2 Workflow of the nuclear spatial pattern analysis pipeline. 1 The pipeline takes a multi-tiff time series stack as input. $\mathbf{2}$ The muscle cell boundary is extracted using FMAj tool. $\mathbf{3}$ The colour channel which contains nuclei is segmented to obtain the nuclear regions. The muscle boundary is used to remove nuclei which lie outside the muscle cell. The external nuclei (present inside the fragments of dead muscle) are identified and removed from the segmentation results to avoid incorrect feature calculations. $\mathbf{4}$ \& $\mathbf{5}$ Nuclear spatial pattern features are calculated using the extracted nuclear regions and these features are used for time series statistical analysis of the myonuclear distributions and localization during metamorphosis
As mentioned before, we require the muscle boundary to remove external nuclei located outside persistent muscles. The color channel of input stack containing muscle cells is used for segmentation. The muscle boundaries are obtained by using an imageJ based muscle analysis tool, FMAj [23]. We also extract morphological features from muscle boundary to understand the relationship between nuclear distribution and muscle mass change.

\section{2) Nuclear Segmentation}

Due to low resolution of images, it is difficult to extract boundaries of nuclei when they are close to each other. For example, a clustered group of nuclei appear as a large blob of bright fluorescence, as shown in Fig. 3b, b'. For this reason, instead of detecting each nucleus, we extract regions where nuclei are located. Each region can contain one nucleus or multiple nuclei. Nuclear segmentation is used to detect myonuclei. To avoid incorrect segmentation due to imaging noise, we first smoothen the image using a bi-exponential edge preserving smoother (BEEPS) [26]. This technique smoothens the high intensity spots near the muscle boundary which occur due to dual channel imaging, while retaining the edge information of nuclei.

For segmentation of nuclei, we use the negative Laplacian of Gaussian (LoG) filter based scheme [27]. The LoG filter has been used previously to find dark circular spots of radius $\sigma$ surrounded by bright backgrounds $[28,29]$. The general idea is that after Gaussian blurring, the intensity distribution of a nuclei form a smooth ridge and the LoG filter can locate the nuclei by detecting the peak point of these ridges. We use a negative LoG kernel (i.e., $-L_{\sigma}$ ) to enhance bright nuclei surrounded by a dark background. Here, $\sigma=6$ which is the average radius of myonuclei in our dataset, is used. In our images, the LoG filter increases the intensity of the regions where nuclei are present. We achieve nuclear segmentation by
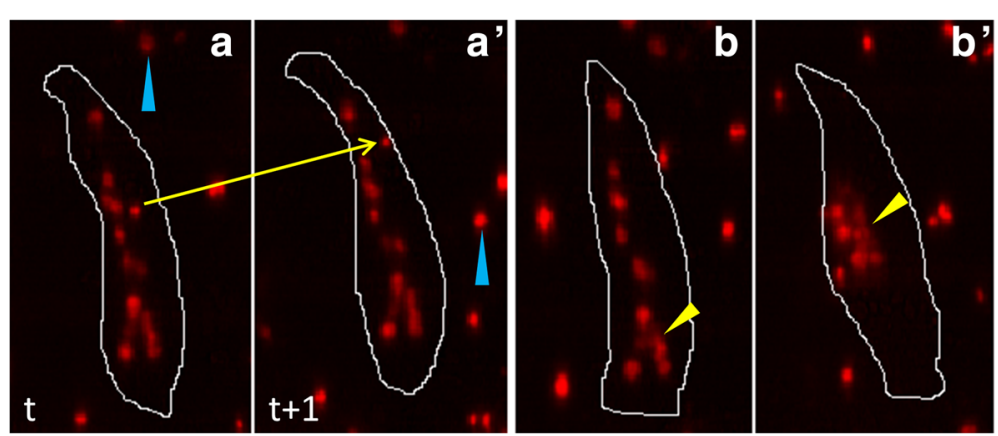

Fig. 3 a Examples of internal and external nuclei. b Examples of nuclear clustering. a The arrow show the movement of an external nucleus over a muscle cell between time points $t(\mathbf{a})$ and $t+1\left(\mathbf{a}^{\prime}\right)$. The rest of the slow moving nuclei inside the muscle cell are internal nuclei. The cyan arrowheads show external nuclei located outside muscle cells. $\mathbf{b} \& \mathbf{b}^{\prime}$ The yellow arrowheads show the clumping of nuclei in two different muscle cells 
applying binary thresholding to the filtered image. Any pixel of intensity 255 was labelled as nuclear region whereas the rest of the pixels were treated as background. Figure 4 shows the results of different stages of nuclear segmentation. Smoothening of image results in decrement in the number of false positives during segmentation (Table 1). Also, by using the LoG filter as pre-processing step, we improve the segmentation of nuclei which have relatively low intensity as compared to the others.

Since we only want the nuclei present inside the muscle cells for analysis; after obtaining the segmentation results, the external nuclei located outside the muscle boundary are removed, as shown in Fig. 4e. In addition, we have to remove the external nuclei that appear inside muscles as a result of overlapping in image projections. We will describe the classification technique used for removing external nuclei in the next section.

\section{3) Nuclear Tracking and Classification}

In order to remove the external nuclei from the segmentation results, we designed a new methodology to classify external and internal nuclei based on their movements. There is a significant difference in the movement of these two types of nuclei. The external nuclei move faster $(25-37 \mu \mathrm{m} / \mathrm{h})$ than internal nuclei $(0-12 \mu \mathrm{m} / \mathrm{h})$. In our classification methodology, we use such a motion characteristic to differentiate between external and internal nuclei. First, we obtain the tracks of nuclei based on a proximity criterion and then classify these tracks on the basis of a cost function derived from nuclear movement in each track.

Various studies were done on tracking nuclei previously [30-32]. However, these problems were customized for tracking nuclei during cell division. On the other hand muscles have multiple nuclei and they don't undergo division. Combined with various issues in our dataset like indistinguishable nuclei due to their adhering to each other, missing nuclei in many time points due to movement of muscles during imaging, etc.; makes it a unique problem which cannot use the previous nuclei tracking techniques. In the followings, we discuss our approach on nuclear tracking and classification in detail.

Step 1: Generating tracks of nuclei First, we use connected component analysis on the nuclear regions extracted in previous section, to detect and label blobs [33, 34]. Each blob can contain one nucleus or multiple nuclei. We use these labelled nuclei to generate tracks. However, in our dataset, it is impossible to track a nucleus from beginning to the end of time series. As mentioned before, at certain time points the nuclei are so close to each other that it is difficult to distinguish them, and due to this issue, we get incomplete tracks of nuclei. Therefore, instead of trying to track nuclei throughout the development, we generate multiple smaller tracks. For example, we are tracking a nuclei $n_{1}$ and the track number is $k_{1}$. At a time point $T=t, n_{1}$ comes close to another nucleus/group of nuclei and form a large clump of high intensity (individual nucleus not visible). At time point $T=t+1$, a nucleus separates from the group of nuclei. However, we are not sure whether it is nucleus $n_{1}$ or other nucleus from the group. In order to avoid this discrepancy, we call this separated nucleus $n_{2}$ and create a new track $k_{2}$. In this manner, we create multiple tracks of the same nucleus over a period of time. The tracks are created based on the nearest neighbour approach. For a nucleus at time point $t$, its nearest neighbour at time point $t+1$ is found using the minimum distance between centroids of nuclei as criterion. Similarly, for each nucleus at time point $t+1$, its nearest neighbour at time point $t$ is found. We observed three types of relationships between nuclei in adjacent time points as shown in Fig. 5a, a'.

Case I: Nucleus $n 1$ from time point $t$ is the nearest neighbour of nucleus $n 1^{\prime}$ from time point $t+1$ and nucleus $n 1^{\prime}$ from time point $t+1$ is the nearest neighbour of nucleus $n 1$ from time point $t$. Therefore, they have a

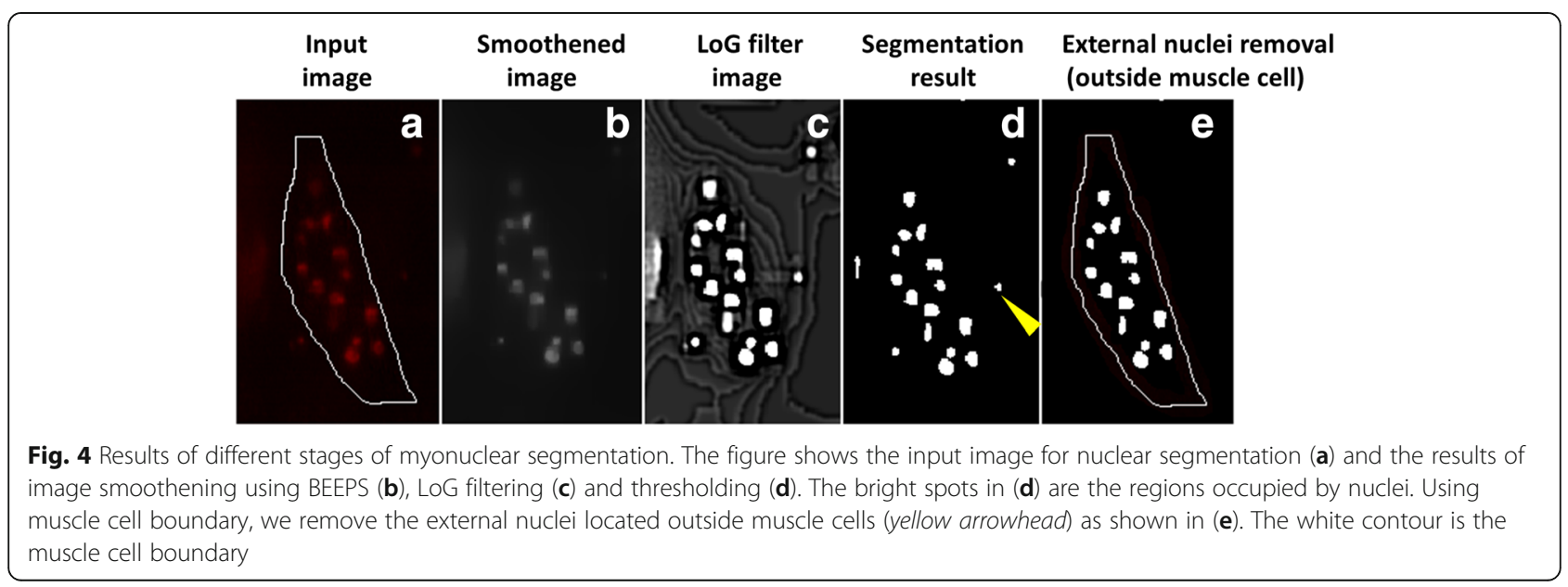


Table 1 Nuclear segmentation performance evaluation

\begin{tabular}{llllll}
\hline & True positive & False positive & False negative & False positive rate & False negative rate \\
\hline Otsu thresholding without LoG filter & 846 & 0 & 108 & 0 & 0.11 \\
LoG based segmentation with BEEPS smoothening & 1180 & 29 & 0 & 0.024 & 0 \\
LoG based segmentation without BEEPS smoothening & 1180 & 334 & 0 & 0.221 & 0 \\
\hline
\end{tabular}

mutual relationship, indicating that these two nuclei are the same.

Case II: Nucleus $n 1$ from time point $t$ is the nearest neighbour of nucleus $n 2$ from time point $t+1$, but nucleus $n 2$ is not the nearest neighbour to nucleus $n 1$. This indicates that $n 1$ is a group of nuclei instead of single nucleus and $n 2$ is a nucleus that has broken off from group of nuclei $n 1$ (Fig. 5a). Here, 'broken off' refers to separation of nuclei which are very close to each other.

Case III: This is the opposite of case II. Nucleus n1' from time point $t+1$ is the nearest neighbour to nucleus $n 2$ from time point $t$; however, nucleus $n 1^{\prime}$ is not the nearest neighbour of nucleus $n 2$ (Fig. 5a'). This would indicate that $n 1^{\prime}$ is a group of nuclei that was formed by joining nucleus $n 2$ with another nucleus. Here, joining refers to two or more nuclei adhering to each other, making them indistinguishable.

Next, we explain the approach used to generate tracks from the relationships between nuclei in adjacent time points. At the first time point of time series stack, every nucleus starts a new track. If a nucleus has mutual relationship with another nucleus from the next time point (Case I), the track continues. However, if a nucleus has one way relationship with a nucleus in the next time point (Case III), its track terminates. New tracks are created when a nucleus does not have a mutual relationship with any nucleus in previous time point (Case II) (Fig. $\left.5 \mathrm{a}, \mathrm{a}^{\prime}\right)$. We represent the tracks in the form of a $\mathrm{T}$ by $\mathrm{X}$ table; where $\mathrm{T}$ is the time point and $\mathrm{X}$ is the total number of tracks. It contains the labels of nuclei. Each nucleus in a time point has a unique label. These unique labels are generated by finding connected components in the image (binary image containing nuclear region as 255 and background as 0 ) and labeling each component/ nuclear region. The labels are assigned based on the location of nuclei along the y axis. Therefore, if there is a difference in the sequence of nuclei along y axis in subsequent time points, the same nuclei will have different labels. If external nuclei are also present, the labels of nuclei change. Track 2 in Additional file 1: Table S1 has

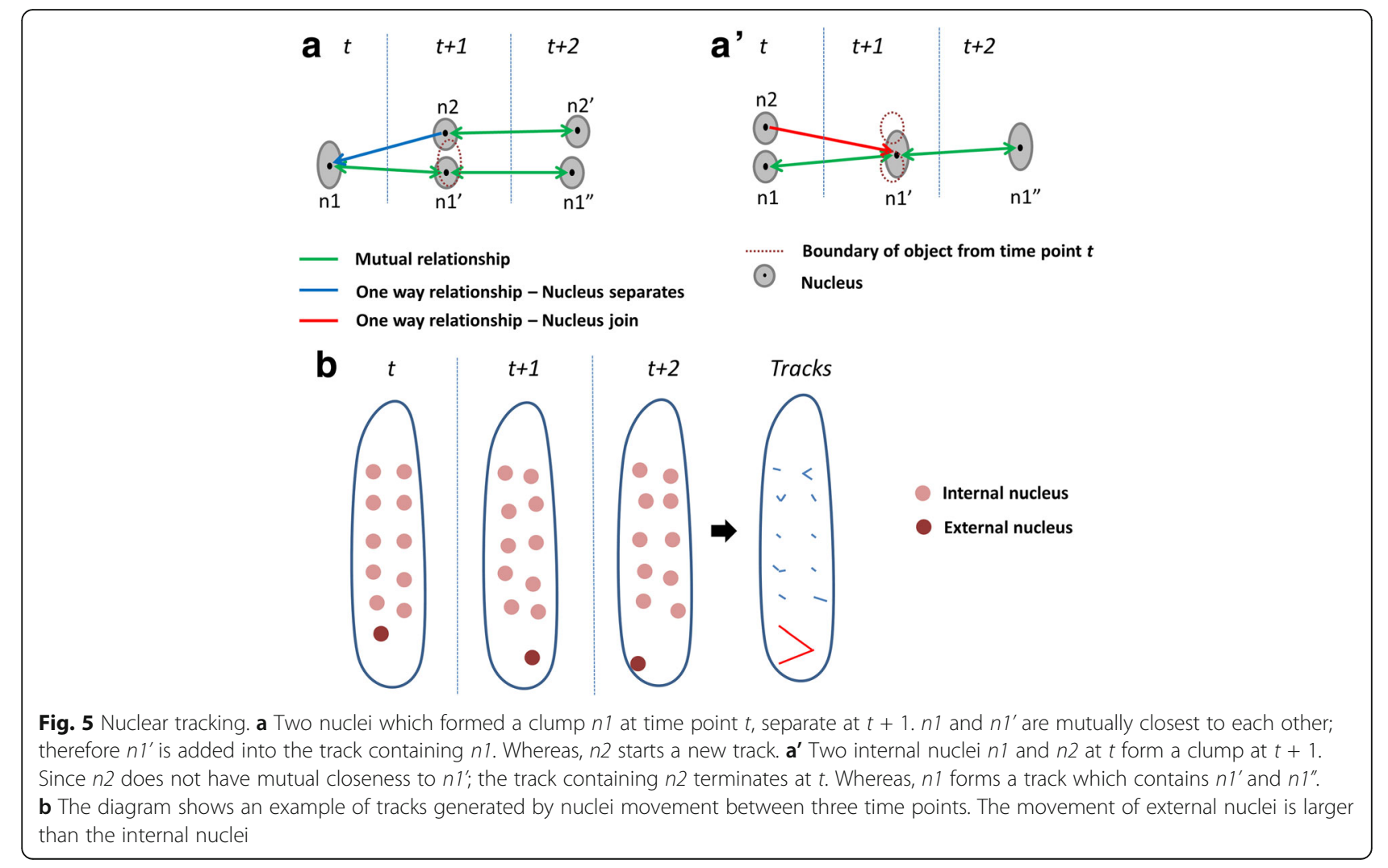


different labels at many time points for the same nucleus. In the case of track 1 , the nucleus is closest to $x$ axis and there are no nuclei in its proximity; therefore its label does not change in subsequent time points.

We are able to track the nuclei accurately when their movement between adjacent time points is close to zero. However, that is not the case throughout pupal development. The movement of muscle cells varies during the development of pupa. Between 12 and $40 \mathrm{~h}$ after head eversion, due to fast movement of muscle cells, the distance covered by internal nuclei in adjacent frames is high ( $>10$ pixels/12.4 $\mu \mathrm{m}$ distance between centroids of muscle cell in consecutive time points). Between 40 to $90 \mathrm{~h}$ after head eversion, muscles move slowly. In order to correctly track the nuclei between two consecutive time points, we adjust the position of nuclei at one of the time points to compensate the movement of muscle cells. First, we find the amount of displacement muscle cell undergo, by measuring the displacement of its centroid in two consecutive time points. For example, centroid of muscle cell moved $x$ pixels horizontally and $y$ pixels vertically between time point $t$ and $t+1$. Next, we translate the image at time point $t$ horizontally by $x$ pixels and vertically by $y$ pixels, so that the nuclei at time point $t$ and $t+1$ align with each other. This alignment facilitates the tracking process by reducing the number of incorrect matching of nuclei between consecutive time points.

Step 2: Distinguishing internal from external nuclear tracks The movement of internal nucleus is much slower than the movement of external nucleus. We exploit this property to classify these two types of nuclei. Intuitively, if a track belongs to an external nucleus, then the average movement of nucleus between consecutive frames should be higher as compared to a track which belongs to an internal nucleus. A schematic diagram in Fig. 5b shows the difference between the track of an internal and external nucleus. Therefore, we design a cost function which is an indicator of the nuclei motion. For a track $x$ of length $n_{x}$ which starts at time point $t_{s}$ and ends at time point $t_{e}$, the cost function $M(x)$ is given as

$$
M(x)=\frac{1}{n_{x}} \sum_{i=t_{s}+1}^{i=t_{e}} D_{i} *\left(1-O_{i}\right)
$$

where $D_{i}$ is the distance between centroid of nuclei in consecutive time points and $O_{i}$ is the percentage overlap between nuclei in consecutive time points. Higher value of cost function indicates higher possibility of the track belonging to an external nucleus and vice versa. The overlap factor $\left(O_{i}\right)$ increases the cost when the overlap between nuclei is low.
After obtaining the cost function for every track, we classify them based on a cost cut-off. Tracks whose cost function is higher than a threshold $\lambda$ are classified as tracks of external nuclei. The external nuclei from these tracks are removed from the segmentation results (Fig. 6). Since, the position of nuclei is normalized according to the movements of muscle cells during calculations; the threshold $\lambda$ should be same for every time series stack. We obtain the value of threshold by performing accuracy tests on training datasets, which will be discussed in the results section.

\section{Nuclear spatial pattern feature}

During certain time points, it is impossible to identify the location of each nucleus because multiple nuclei adhere to each other and form a large clump of high intensity. In these cases, extraction of nuclear region generates a blob (connected components) which contains many nuclei. For this reason, we cannot use point pattern analysis [18] to study nuclear distribution, but have to design a different approach which uses complete nuclear structure inside muscles as compared to only centroids for nuclear pattern feature generation. We design three new features which quantify different types of nuclear distributions, i.e. nuclear spatial density index, longitudinal nuclear spread and lateral nuclear spread. Before analysing the nuclear distribution, we rearrange the nuclear structure along the straightened medial axis of the muscle cell.

\section{Rearrangement of nuclei in straightened muscle cell}

Aligning the nuclei along the straightened medial axis of muscle cell helps to provide a spatial reference for comparing nuclei from different samples. We assume that the medial axis of muscle cell is straight and adjust the position of nuclei according to the changes in the curvature of the medial axis (Figs. $7 \& 8 \mathrm{a}-\mathrm{a}$ "). The straightening algorithm is as follows:

1) Find the centroid of a nucleus.

2) Find the smallest distance $(d x)$ to the centroid from the medial axis of muscle cell. Let the point at the medial axis which has the least distance from the centroid be $m$.

3) Find the length of medial axis $(d y)$ between the start of medial axis and point $m$.

4) Find the angle $(\alpha)$, i.e. the angle between the tangent at point $m$ of medial axis and y axis.

5) Assuming that the start point of straightened medial axis lies at $x=\mathrm{X}, y=0$, then the new coordinate of point $m$ is $P(X, d y)$ and the centroid of the nucleus is $P(X \pm d x, d y)$. $\mathrm{X}$ is a constant number such that $X \pm d x$ is never negative. The sign in $X \pm d x$ 

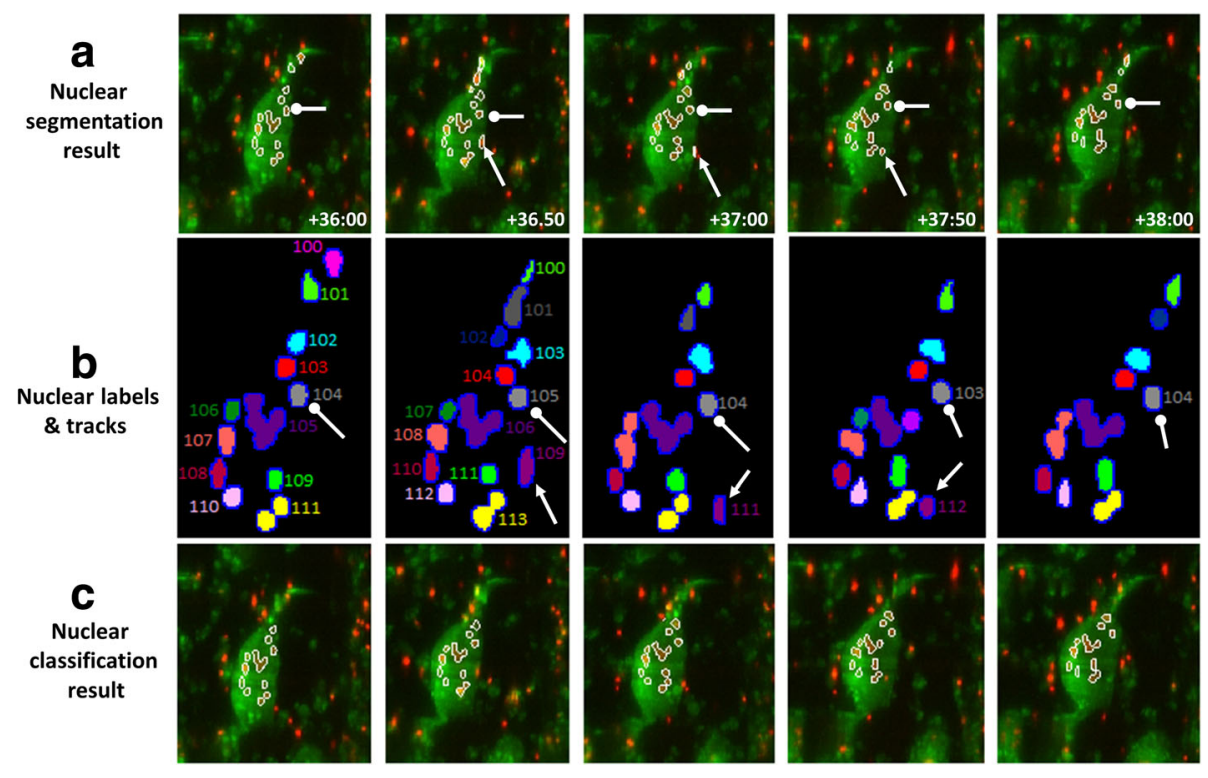

Fig. 6 Results of nuclear classification. a The images show the result of nuclear segmentation for five time points selected from a pupa expressing Atg9 RNAi. DIOM muscle from 3rd segment of pupa abdomen was used for this analysis. The segmented nuclei contain both internal and external nuclei. The white arrow and oval arrow indicates an external and internal nucleus. $\mathbf{b}$ The labels of nuclei at each time point are shown. Nuclei which have same colour in subsequent time points belong to same track. The details of nuclear tracks are shown in Additional file 1: Table S3. c The images show results of nuclear classification. The contours of classified external nuclei are removed from the segmentation results

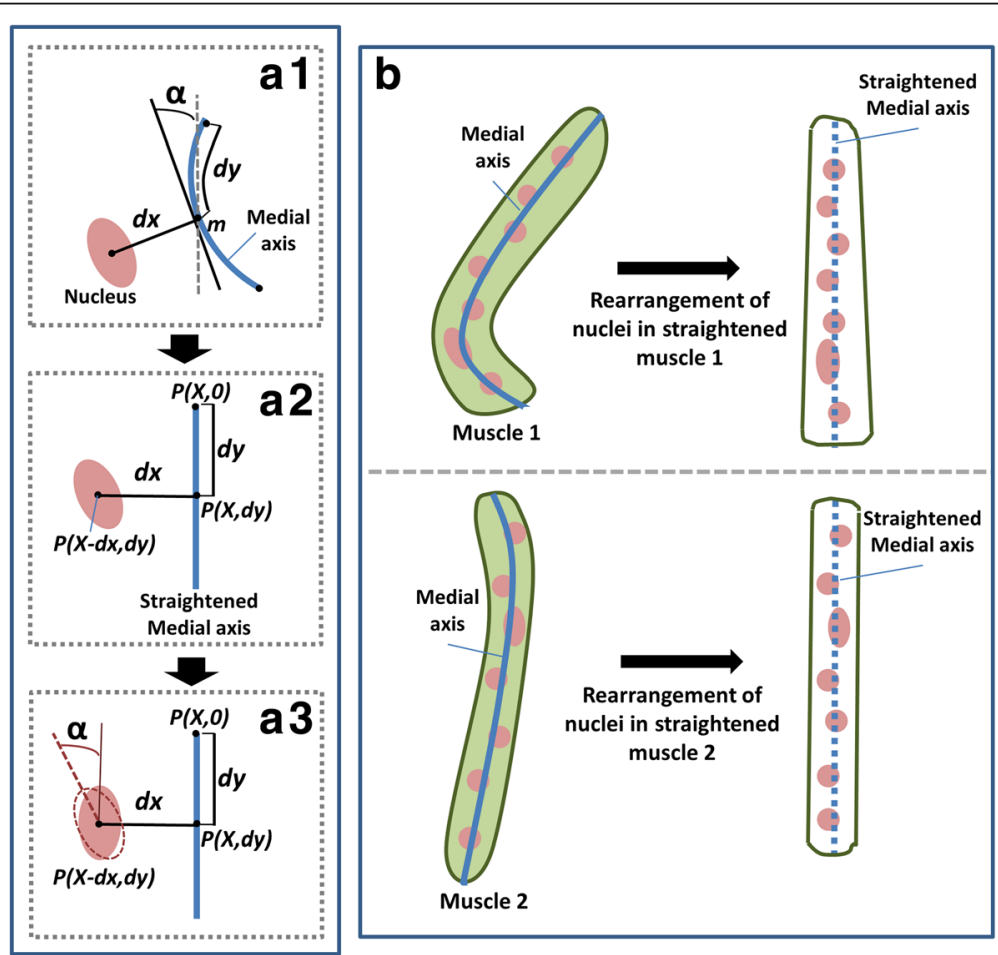

Fig. 7 Rearrangement of nuclei in straightened muscle cell for comparing nuclei from different samples. a1-a3 The figure shows various steps involved in rearrangement of a nucleus along straightened muscle medial axis i.e. calculation of new coordinates of nucleus and medial axis (a1), translation of nucleus to new coordinate (a2) and rotation of nucleus about its centroid (a3). b Rearranged nuclei along medial axis are shown for two muscles with different shapes 

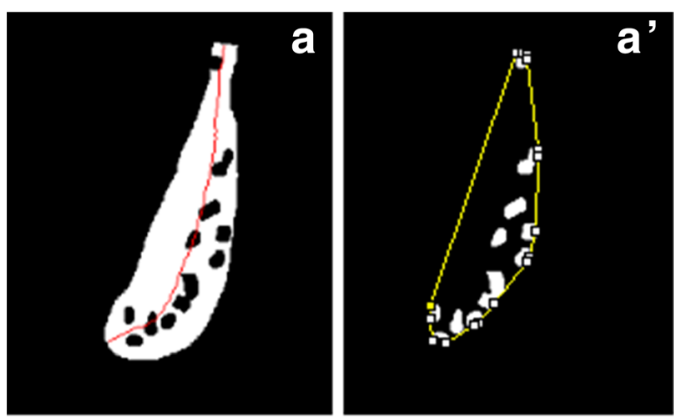

NSD $=0.77$

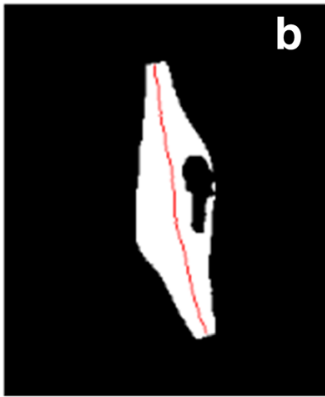

NSD $=0.11$

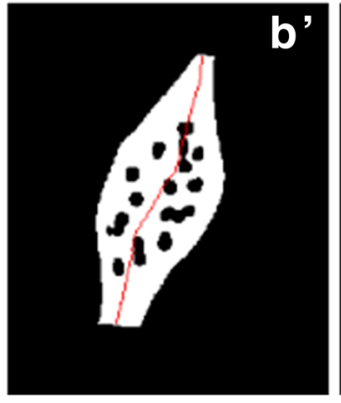

NSD $=\mathbf{0 . 3 8}$

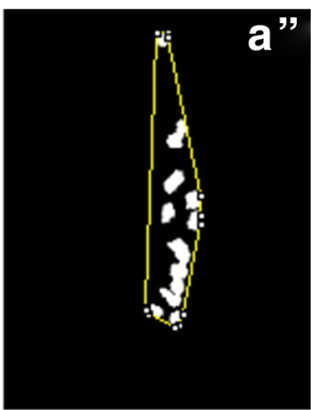

NSD $=0.52$

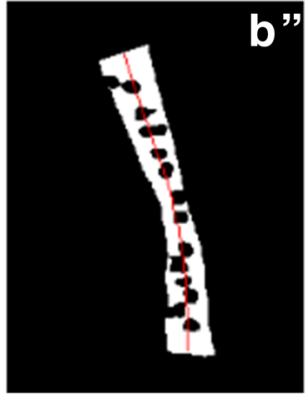

NSD $=\mathbf{0 . 7 2}$

Fig. 8 Example showing differences in nuclear spatial density due to change in cell size. (a-a") NSD: Nuclear Spatial density. The figure shows straightening of nuclei with respect to the medial axis of nuclei. It compares the nuclear spatial density index for straightened (a") and un-straightened nuclei $\left(\mathbf{a}^{\prime}\right)$. $\mathbf{b}-\mathbf{b}^{\prime \prime}$ The value of nuclear spatial density is lower for densely packed nuclei $(\mathbf{b})$ as compared to evenly distributed nuclei $\left(\mathbf{b}^{\prime \prime}\right)$

depends on whether the nucleus is located on left or right side of medial axis.

6) Next, Translate the nucleus to point $P(X \pm d x, d y)$ and rotate the nucleus by an angle $\alpha$.

7) Repeat steps 1-6 for each nuclei inside the muscle cell.

1) Nuclear spatial density

As mentioned earlier, it has been previously shown that there is an association between nuclear localization and muscle mass change. To confirm this hypothesis, we design a feature that measures the nuclear spatial density with respect to the cell size.

Nuclear spatial density $(\mathrm{NSD})=\frac{\text { Area of convex hull of nuclei }}{\text { Area of muscle cell }}$

A straightened nuclear structure is used to calculate the convex hull. The convex hull of a nuclear region is the smallest convex set which contains that region [35]. If nuclear spatial density is close to 1 , the nuclei are located close to the muscle boundary and are distributed more evenly. Alternatively, a low value of NSD indicates that the nuclei formed a cluster and occupied a small part of the muscle cell. In Fig. 8, the sample $b$ has the smallest NSD, resulting from clustering of the nuclei. In sample $b$, although the nuclei are more spread out, the NSD is not as high as in sample $b$ ". This is because sample $b^{\prime}$ has a larger cell size than sample $b^{\prime \prime}$.

\section{2) Longitudinal nuclear spread}

Longitudinal nuclear spread characterizes the polar and anti-polar migration of nuclei. It is defined as the distance between the extremes of the nuclei along the medial axis and denoted as $L_{n}$. The normalized migration of nuclei along the medial axis/normalized longitudinal nuclear spread $N M_{\text {lon }}$ is defined as:

$$
N M_{l o n}=L_{n} / L_{c}
$$

where $L_{n}$ is the longitudinal nuclear spread and $L_{c}$ is the length of the muscle cell (Fig. 9a). A high $N M_{\text {lon }}$ indicates that the nuclei are close to the poles of the muscle cell and a low value indicates that they are far.

\section{3) Lateral nuclear spread}

Lateral nuclear spread characterizes the movement of nuclei away from the medial axis of muscle during late stages of pupal development. The lateral nuclear spread $M_{\text {lat }}$ is defined as: 


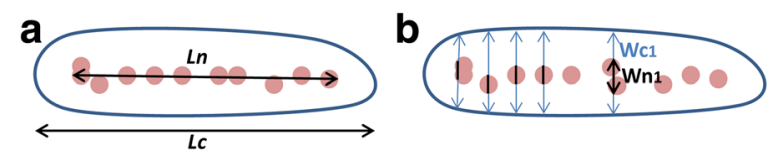

Fig. 9 Schematic diagram explaining the derivation of nuclear pattern features. a Normalized longitudinal nuclear spread. b Normalized lateral nuclear spread

$$
M_{\text {lat }}=\frac{1}{u} \sum_{i=1}^{i=u} W n_{i}
$$

where $W n_{i}$ is the width of the nuclear structure at the $i^{t h}$ location on the medial axis, and $u$ is the number of samples taken along medial axis (Fig. 9b). All of the samples are collected at equal interval along medial axis. A high $M_{\text {lat }}$ indicates an increase in the distance between nuclei along the width of muscle i.e. two-row formation of nuclei.

In order to quantify the influence of muscle mass change on lateral displacement of nuclei, we also designed normalized lateral nuclear spread. Normalized lateral nuclear spread $N M_{\text {lat }}$ is defined as:

$$
N M_{\text {lat }}=\frac{1}{u} \sum_{i=1}^{i=u} W n_{i} / W c_{i}
$$

where $W c_{i}$ is the width of muscle cell at the $i^{\text {th }}$ location on the medial axis. A high $N M_{\text {lat }}$ indicates that the nuclei are close to the boundary of the muscle cell. A few examples of the values of longitudinal nuclear spread and lateral nuclear spread are shown in Fig. 10.

\section{Implementation of algorithm}

The nuclear pattern analysis algorithms were implemented in Java and incorporated as a part of the FMAj tool. We have used two external libraries: Mexican hat filter [27] and hull and circle plugin [35]. The nuclear analysis module in FMAj is divided into three sections: nuclear segmentation, nuclear classification and nuclear feature generation. Nuclear segmentation is performed on complete image. Whereas, nuclear classification is performed on nuclei inside muscles using muscle boundaries. The nuclear features generated by FMAj are stored in a MySQL database. The analysis of the features was done in excel and FMAj.

\section{Results}

Evaluation of nuclear segmentation

We evaluated the performance of segmentation at object level rather than pixel level. We manually counted the false negative, false positive and true positive by comparing the segmentation results with original image. False positive (FP) is the count of segmented objects which were not nuclei. True positive (TP) is the count of segmented objects which were nuclei. False negative (FN) is the count of nuclei which were not segmented. We evaluated the segmentation results for 140 time points that were selected from two Atg9 samples. We used two parameters for evaluation:

$$
\begin{aligned}
& \text { False negative rate }=\mathrm{FN} /(\mathrm{FN}+\mathrm{TP}) \\
& \text { False positive rate }=\mathrm{FP} /(\mathrm{FP}+\mathrm{TP})
\end{aligned}
$$

The false negative rate is the ratio of false negative and the total number of actual nuclei; whereas, the false
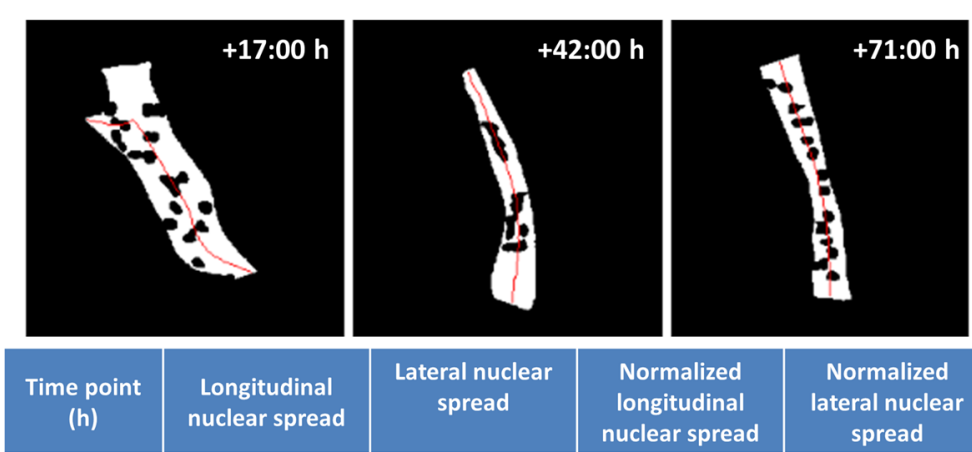

Normalized

spread

longitudinal nuclear spread

lateral nuclear spread

\begin{tabular}{|l|l|l|l|l|}
\hline+17 & 129 & 14.2 & 0.76 & 0.32 \\
\hline+42 & 105 & 7.53 & 0.59 & 0.36 \\
\hline+71 & 157 & 6.76 & 0.87 & 0.30 \\
\hline
\end{tabular}

Fig. 10 Comparison of nuclear pattern features of a muscle at three different time points. The muscle shown in the figure is from a control samples. At $+71 \mathrm{~h}$, the muscle has highest $M_{\text {lon }}$ and $N M_{\text {Ion }}$ because the nuclei are closer to the poles of the muscle cell than at other time points. Whereas, the muscle has highest $M_{\text {lat }}$ at $+17 \mathrm{~h}$; because the nuclei are more spread out along the width of muscle. The value of $N M_{\text {lat }}$ is approximately similar at three time points. This indicates that at these three time points, the spread of nuclei with respect to width of muscle is similar 
positive rate is the ratio of false positive and the total number of nuclei segmented.

The performance of thresholding segmentation with LoG filtering is compared with thresholding (Otsu) without LoG filtering in Table 1. As shown in Table 1, LoG based method has zero false negative rate and 0.024 false positive rate. It means that LoG based method correctly segments every nuclei; however it also segments some non-nuclei high intensity spots (imaging noise). In comparison, Otsu thresholding without LoG filtering has false negative rate of 0.11 which is not suitable for spatial pattern analysis. The results of LoG based segmentation without bi-exponential smoothening is also shown in Table 1 . The false positive rate is higher in the case of segmentation without smoothening as compared to with smoothening. This verifies that smoothening of our images is necessary to remove imaging noise.

\section{Evaluation of nuclear classification}

$\lambda=7$ is used for nuclei classification in our dataset. In order to find the correct $\lambda$ for our dataset, we used a training dataset to calculate the performance of classification for different thresholds. We calculated following parameters for classification performance evaluation: accuracy, false positive rate and false negative rate.

$$
\text { Accuracy }=(\mathrm{TP}+\mathrm{TN}) /(\mathrm{TP}+\mathrm{FP}+\mathrm{TN}+\mathrm{FN})
$$

We measured the false negative, false positive, true negative and true positive by comparing the classification results with a ground truth which was generated manually using FMAj [23]. False positive (FP) is the count of external nuclei which were falsely classified as internal nuclei. True positive (TP) is the count of correctly classified internal nuclei. False negative (FN) is the count of internal nuclei falsely classified as external nuclei. True negative (TN) is the count of correctly classified external nuclei. The performance evaluation was done for 6 different muscle cells from different genotypes i.e. two samples from control, Atg9 and Atg12; data was generated from at least 50 time points per muscle cell. We visualized accuracy, false positive rate and false negative rate for these 6 muscles in a graph as shown in Fig. 11a-c. The threshold at which accuracy was highest and error rates were minimum was selected for classification of nuclei (Shown by black dotted line) i.e. $\lambda=7$.
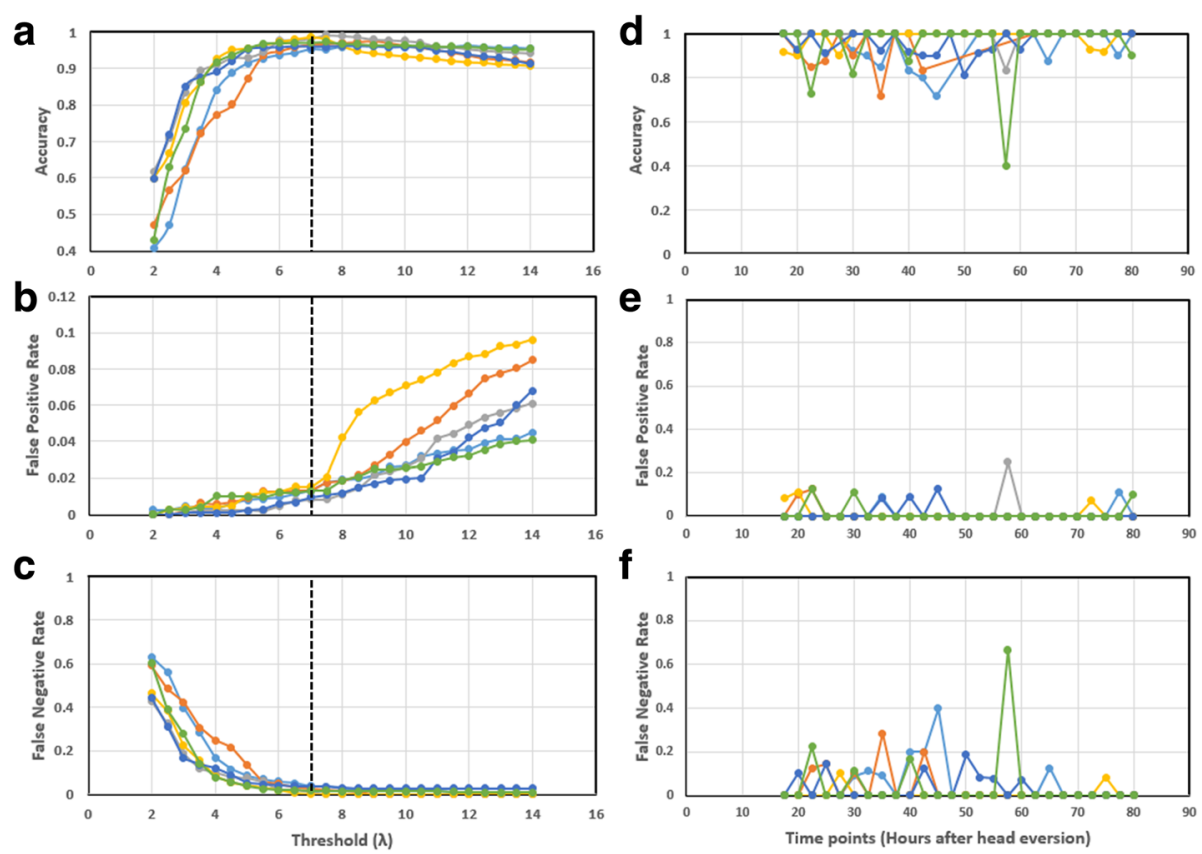

$$
\rightarrow \text { Muscle } 1 \rightarrow \text { Muscle } 2 \rightarrow \text { Muscle } 3 \rightarrow \text { Muscle } 4 \rightarrow \text { Muscle } 5 \rightarrow \text { Muscle } 6
$$

Fig. 11 Performance evaluation of nuclear classification at different threshold values and different development stages. a-c The graphs compare the performance of nuclear classification at different thresholds using following parameters i.e. accuracy (a), false positive rate (b) and false negative rate (c). Each series in the graph represents a different muscle cell. The black dotted line indicate the threshold value at which accuracy is highest and error rates are lowest. This threshold value is used for nuclear classification. $\mathbf{d}$-f The graphs compare the performance of nuclear classification at different stages of pupal development using following parameters i.e. accuracy (d), false positive rate (e) and false negative rate (f). Large movements of internal nuclei results in low accuracy and high error during between +20 and $+50 \mathrm{~h}$. In comparison, accuracy is high after $+50 \mathrm{~h}$ due to slow movement of internal nuclei 
Thus, we measured the performance of our nuclear classification algorithm at $\lambda=7$, for the same 6 muscles which were used for calculation of $\lambda$. The results are as follows: average accuracy $=96.9 \pm 1.2 \%$, average false positive rate $=1.2 \pm 0.2 \%$, average false negative rate $=2.1 \pm 1.3 \%$. A comparison of nuclear classification performance evaluation during different stages of pupal development is shown in Fig. 11d-e. It can be observed that the accuracy of nuclear classification suffers due to the large movements of internal nuclei between $20 \mathrm{~h}$ and $50 \mathrm{~h}$ after head eversion. Whereas, during later stages of pupal development, the reduction in movement of internal nuclei results in high accuracy and low false positive and negative rates.

\section{Results of Myonuclear spatial pattern analysis}

We previously described the effects of genetic perturbations on nuclear migration remodeled muscles [14]. Here, we used the nuclear spatial pattern analysis algorithm to quantify nuclear distribution in $C p 1^{\text {shRNA }}$, Atgs ${ }^{\text {shRNA }}$ and control muscles. To compare different genotypes, we calculated the nuclear features for each genotype and performed a non-parametric Mann-Whitney $\mathrm{U}$ test. The significance test was performed for nuclear features at every time point. We plotted $-1 * \log _{10}(\mathrm{P}$-val) for each time point where nuclear features had been calculated. Value of $-1{ }^{*} \log _{10}(\mathrm{P}$-val $)$ above $1.3(\mathrm{P}$-val $=0.05)$ is considered significant. We used head eversion (HE) as a temporal reference to compare different samples. Time was represented as hours (h) after head eversion. DIOM muscle from 3rd segment of pupa abdomen was used for this analysis. We have also compared the properties of nuclear tracks between different genotypes like start and end time of track, length of track, nucleus speed etc. (Additional file 1: Table
S2). It shows that external nuclei display high speed and large movements compared to internal nuclei irrespective of genotype.

\section{Cp1 participates in the anti-polar/polar migration of nuclei}

Quantitative nuclear pattern analysis confirmed that the knockdown of $C p 1$ affected anti-polar nuclear migration in early pupation (Additional file 1: Figure S1c, d). In control muscles, anti-polar migration reduced the expanse of nuclei along the medial axis $\left(L_{n}\right)$ by $\sim 97.5 \mu \mathrm{m}$ between $+28.9 \mathrm{~h}(205.8 \mu \mathrm{m})$ and $+46.6 \mathrm{~h}(108.4 \mu \mathrm{m})$ (Additional file 1: Table S3, Fig. 1a, b). By contrast, $C p 1$ silencing resulted in reduction of $L_{n}$ by $\sim 58.6 \mu \mathrm{m}$ between $+23.5 \mathrm{~h}(209.8 \mu \mathrm{m})$ and $+54.1 \mathrm{~h}(151.3 \mu \mathrm{m})($ Fig. 12a). Unlike control, where nuclear polar migration increased the value of $L_{n}$ by $\sim 82.6 \mu \mathrm{m}$ between $+46.6(108.4 \mu \mathrm{m}) \mathrm{h}$ and +72.1 h $(190.9 \mu \mathrm{m})$; $C p 1$ RNAi did not show polar migration. Due to decreased anti-polar migration in $C p 1$ RNAi, the values of $L_{n}$ are significantly different for control and Cp1 RNAi between $+42 \mathrm{~h}$ and $+52 \mathrm{~h}$ (Fig. 13a, b).

The normalized longitudinal nuclear spread $\left(N M_{l o n}\right)$, indicated that the reduction in expanse of nuclei along medial axis of muscle $\left(L_{n}\right)$ in $C p 1$ RNAi is due to shortening of muscle cells. This phenomenon was confirmed by very small change in values of $N M_{l o n}$ for $C p 1$ RNAi between $+32.5 \mathrm{~h}$ and $+60 \mathrm{~h}$ (Fig. 13c). Between $+32.5 \mathrm{~h}$ and $+45 \mathrm{~h}, N M_{\text {lon }}$ values changed only by 0.2 $(0.82 \pm 0.07-0.84 \pm 0.05)$ for Cp1 RNAi as compared to $0.27(0.83 \pm 0.05-0.55 \pm 0.13)$ for control. Similarly, between $+45 \mathrm{~h}$ and $+60 \mathrm{~h}, N M_{\text {lon }}$ values changed only by $0.2(0.84 \pm 0.05-0.85 \pm 0.02)$ for $C p 1$ RNAi as compared to $0.28(0.55 \pm 0.13-0.83 \pm 0.03)$ for control. The significantly high values of $N M_{l o n}$ for $C p 1$ RNAi as compared
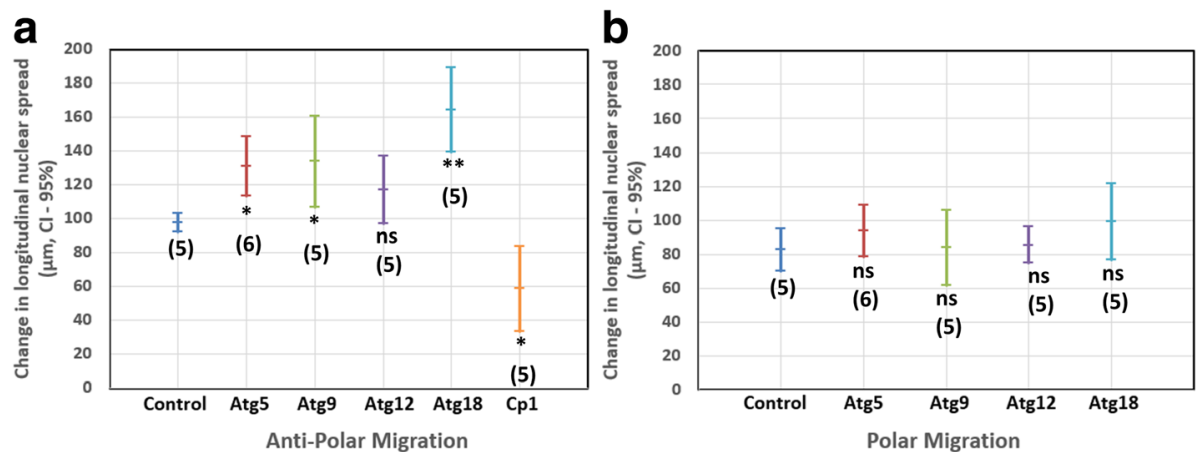

Fig. 12 Comparison of change in longitudinal nuclear spread during polar and anti-polar migration between different genotypes. The graph shows the mean of difference in the longitudinal nuclear spread $L_{n}$ between different genotype for anti-polar migration (a) and polar migration (b). The error bars show the $95 \%$ confidence interval. During anti-polar migration, the movement of nuclei towards the muscle centre is higher in Atg5, Atg9 and Atg 18 compared to control. Therefore, at the end of anti-polar migration the distance between extreme nuclei and poles is significantly large in above mentioned Atg genes. However, during polar migration, the total distance moved by nuclei towards poles in Atgs is not significantly different from control. This would indicate that the reduced expanse of nuclei in Atgs compared to control during polar migration (Fig. 14) is due to positioning of nuclei at large distances from poles at beginning of polar migration. The reduced anti-polar migration in Cp 1 is also shown by the small change in expanse of nuclear structure as compared to control. ${ }^{*} P<0.05,{ }^{* *} P<0.01,{ }^{* * *} P<0.001, \mathrm{~ns}=$ not significant $(P>0.05)$ 

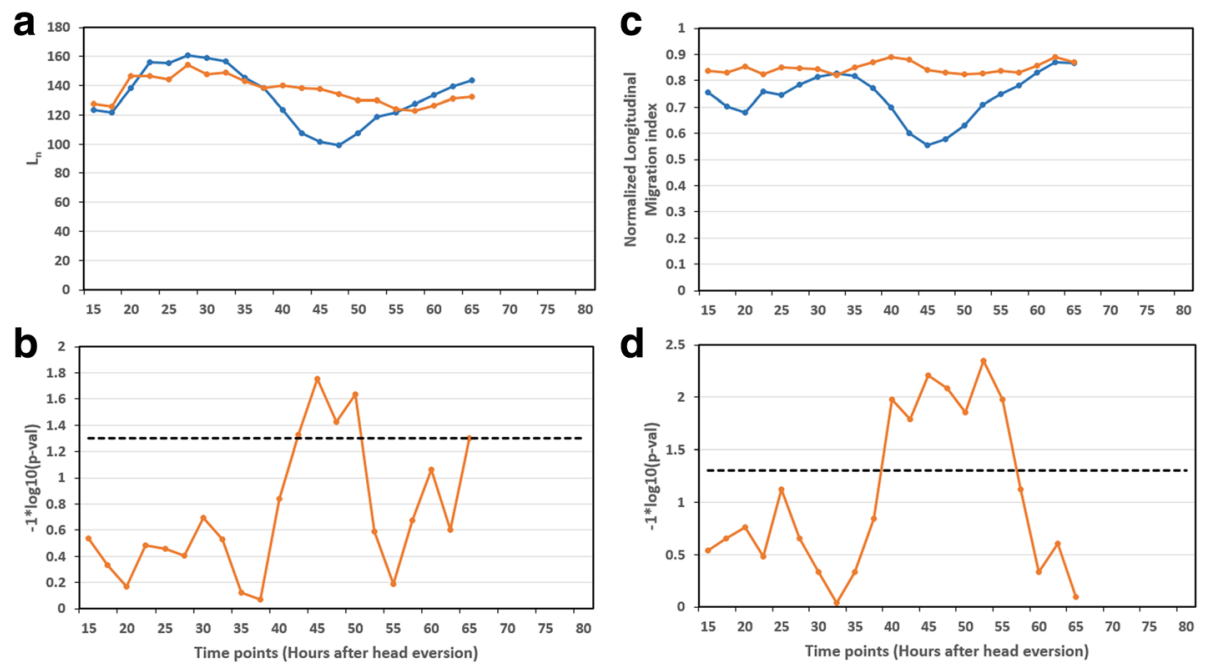

Fig. 13 Longitudinal nuclear spread quantifies the effect of $C p 1$ knockdown on anti-polar migration. a \& c Graphical comparisons of $L_{n}$ (Distance between the extremes of the nuclei along the medial axis) and normalized longitudinal nuclear spread $N M_{\text {lon }}$ between control and Cp1 mutant. Due to absence of anti-polar movement of muscles around $+50 \mathrm{~h}$ in Cpl mutants; the value of both $L_{n}$ and $N M_{\text {lon }}$ is high as compared to control. b \& d Significance graph. For each population, statistics were derived from 5 to 7 DIOM1. The muscles were from segment 3 of abdomen. The graph on top show average values of the features. The horizontal dotted lines in the significance graphs represent the $p$-value 0.05

to control between $+40 \mathrm{~h}$ and $+57 \mathrm{~h}$ indicate that the knockdown of $C p 1$ resulted in absence of anti-polar migration (Fig. 13d).

\section{Silencing of Atg9 and Atg18 increases the anti-polar migration and decreases the polar migration of nuclei} RNAi of Atg5, Atg9 and Atg18 affected myonuclear migration. While knockdown of all Atgs RNAi did not block anti-polar and polar nuclear migration (Additional file 1: Figure S2), the silencing of Atg9 and Atg18 resulted in prolonged anti-polar migration of nuclei $(7.5 \mathrm{~h}$ (median) delay for Atg9 RNAi and $4 \mathrm{~h}$ (median) delay for Atg18) and larger anti-polar movement of nuclei (between $+27.4 \mathrm{~h}$ and $+53 \mathrm{~h}$ for $A \operatorname{tg} 9$ and between $+22.4 \mathrm{~h}$ and $+48.1 \mathrm{~h}$ for $A \operatorname{tg} 18$ ) as compared to control (between $+28.9 \mathrm{~h}$ and $+46.6 \mathrm{~h}$ ) (Fig. $14 \mathrm{a}, \mathrm{b}$ ). The reduction in value of $L_{n}$ during anti-polar migration in Atg5, Atg9, Atg18 and control was $\sim 130.8 \mu \mathrm{m}, \sim 133.9 \mu \mathrm{m}, \sim 164.4 \mu \mathrm{m}$ and $\sim 97.5 \mu \mathrm{m}$; this indicates that nuclei are farther from poles in Atg5, Atg9 and Atg18 as compared to control (Fig. 12a, Additional file 1: Table S3). Knockdown of Atg9 and Atg18 also reduced the polar migration of nuclei (Between $+55 \mathrm{~h}$ and $+72.5 \mathrm{~h}$ for Atg9 and between $+50 \mathrm{~h}$ and $+72.5 \mathrm{~h}$ for Atg18), resulting in larger distances between pole and nearest nucleus (Fig. 14a, b). The value of $L_{n}$ at the end of polar migration in Atg9, Atg18 and control was $\sim 141.6 \mu \mathrm{m}, \sim 162.4 \mu \mathrm{m}$ and $\sim 190.9 \mu \mathrm{m}$ approximately; this indicates reduced polar migration in Atg9 and Atg18 (Additional file 1: Table S3). However, it is important to note that in spite of lower longitudinal nuclear spread in
Atgs, there is no significant difference between the changes in longitudinal nuclear spread (between start and end of polar migration) in Atgs and control as shown in Fig. 12b.

The significant difference in $N M_{\text {lon }}$ between the control and Atg9 and Atg18 RNAi after $+50 \mathrm{~h}$ and $+46 \mathrm{~h}$ respectively proved that the polar/anti-polar migration of nuclei in Atg9 RNAi and Atg18 RNAi is not affected by muscle contraction/elongation (Fig. 14c, d). The effects of silencing Atg5 and Atg12 on nuclear migration are not as prominent as Atg9 and Atg18 silencing. In both Atg5 and Atg12, the values of $L_{n}$ is significantly less than control at the end of polar migration $(\sim 169 \mu \mathrm{m}$ at $+72.1 \mathrm{~h}$ for Atg5, $177.1 \mu \mathrm{m}$ at $+72.2 \mathrm{~h}$ for Atg12 and $\sim 190.9 \mu \mathrm{m}$ for control), although not as low as Atg9 and Atg18; this indicates reduced polar migration (Additional file 1: Table S3). Also, there was no significant difference in the values of $N M_{\text {lon }}$ in Atg5 and Atg12 RNAi during the polar migration. These findings indicate that the decreased polar migration caused by silencing of Atg5 and Atg12 could be due to contraction of muscle cells.

\section{Lateral myonuclear displacement Atg knockdowns correlates with increased diameter of muscles}

As previously reported [14], loss of autophagy resulted in nuclei being arranged in two rows in late metamorphosis as compared to single row formation in control muscles. We used lateral nuclear spread $\left(M_{\text {lat }}\right)$ and normalized lateral nuclear spread $\left(N M_{\text {lat }}\right)$ to quantify the lateral movement of nuclei perpendicular to the medial axis of muscles. In $\operatorname{Atg} 9$ and $A \operatorname{tg} 18 \mathrm{RNAi}$, the value of 

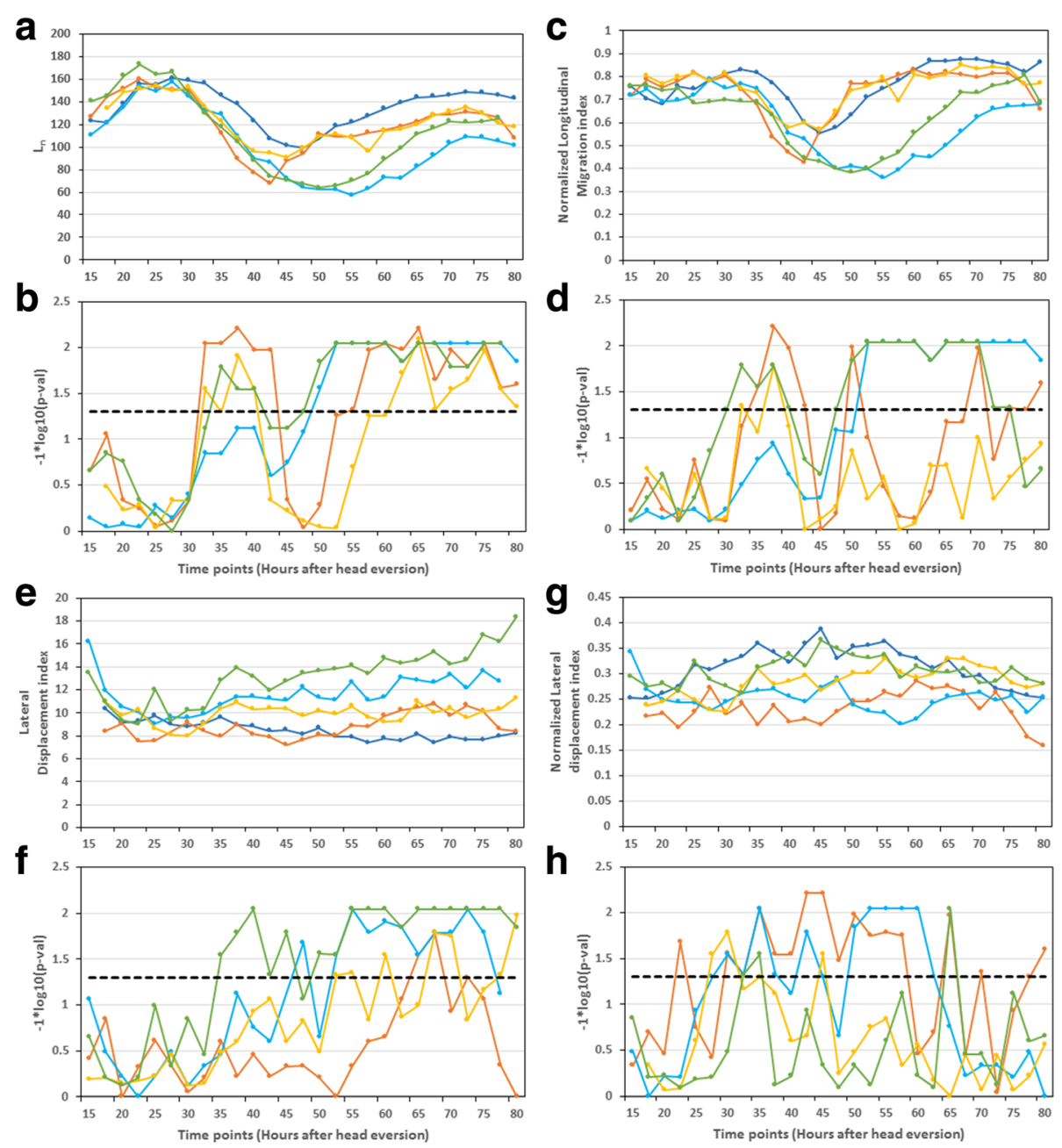

$\rightarrow$ Control $\rightarrow$ Atg5 $\longrightarrow$ Atg9 $\longrightarrow$ Atg12 $\longrightarrow$ Atg18 --- - p-val $=0.05$

Fig. 14 Nuclear pattern features quantifies the effect of loss of autophagy on nuclear distribution. The figure compares following nuclear pattern features y in muscles expressing Atg5 RNAi, Atg9 RNAi, Atg12 RNAi, Atg 18 RNAi and control: (a) $L_{n}$. (c) Normalized longitudinal nuclear spread. e Lateral nuclear spread. $\mathbf{g}$ Normalized lateral nuclear spread. The graphs show average values of the features. $\mathbf{b}, \mathbf{d}, \mathbf{f}, \mathbf{h}$ Significance graph. For each population, statistics were derived from 5 DIOM1 (Segment 3). The horizontal dotted lines in the significance graphs represent the $p$-value 0.05

$M_{\text {lat }}$ was significantly higher in controls after $+53 \mathrm{~h}$ and $+50 \mathrm{~h}$, respectively; suggesting that nuclei moved away from the muscle's medial axis and arranged in a two-row formation (Fig. 14e, f). Interestingly, we did not observe a similar trend in $N M_{\text {lat }}$ for Atg9 and Atg18 RNAi (Fig. 14g). We found the values of $N M_{\text {lat }}$ were significantly lower for Atg9 RNAi compared to controls between $+50 \mathrm{~h}$ and $+70 \mathrm{~h}$ (Fig. 14h). This indicates that between $+50 \mathrm{~h}$ to $+70 \mathrm{~h}$, the nuclei were located away from the muscle cell boundary as compared to control, even though they were arranged in two rows. However, after $+70 \mathrm{~h}$, values of $N M_{\text {lat }}$ were not significantly different between control and Atg9 RNAi. Whereas, in $\operatorname{Atg} 18 \mathrm{RNAi}$, the value of $N M_{\text {lat }}$ were not significantly different from control throughout pupal development. Therefore, the lateral displacement of nuclei might be dependent on the change in muscle width in $\operatorname{Atg} 9$ and Atg18 RNAi. The increase in width of muscle cell due to loss of autophagy could be increasing the distance between nuclei resulting in two-row formation. In case of $\operatorname{Atg} 5$ and $\operatorname{Atg} 12$, the lateral displacement features were unable to statistically prove the two-row formation, despite being confirmed visually, indicating a lower penetration of the phenotype.

The anti-polar/polar migration and lateral displacement of nuclei affect the spatial density of nuclei in muscles

During early stages of pupal development, differences in spatial density of nuclei were observed between Atgs (Atg5, Atg9 \& Atg18) and control. In Atg5 RNAi, significant reduction in NSD was observed between $+25 \mathrm{~h}$ to $+42.5 \mathrm{~h}$ compared to control. This observation could be the result of larger anti-polar migration and smaller lateral 
displacement of nuclei with respect to width of muscle. Significant reduction in spatial density of nuclei was also observed in $\operatorname{Atg} 9$ (between $+30 \mathrm{~h}$ to $+40 \mathrm{~h}$ ) and $\operatorname{Atg} 18$ (between $+35 \mathrm{~h}$ to $+40 \mathrm{~h}$ ). We also observed that the spatial density of nuclei with respect to the muscle area in $\operatorname{Atg} 9$ and Atg18 was significantly lower than the control between $+50 \mathrm{~h}$ to $+70 \mathrm{~h}$ and $+50 \mathrm{~h}$ to $+65 \mathrm{~h}$ respectively (Fig. 15a, b). It indicates that the knockdown of both Atg 9 and Atg18 increased the area devoid of nuclei in muscles. Since, the lateral displacement of nuclei increases between $+50 \mathrm{~h}$ to $+70 \mathrm{~h}$ in Atg9 and Atg18 RNAi; the reduced polar migration could be the reason for low spatial density of nuclei (NSD) with respect to the muscle area. As opposed to other Atgs, Atg12 knockdown did not affect the spatial density of nuclei with respect to the muscle area despite the two-row formation. Increased polar migration during later pupal development could be one of the reasons behind the control like nuclear spatial density in Atg5 and Atg12 RNAi as compared to Atg9 and Atg18 (Fig. 14a, b).

\section{Discussion}

We previously found that muscle atrophy in remodelled muscles is accompanied by extensive myonuclear migration [14]. To better understand the process of nuclear migration in muscle cells, we designed an algorithm to extract a set of nuclear spatial pattern features. Apart from the semi-automated muscle segmentation, all the

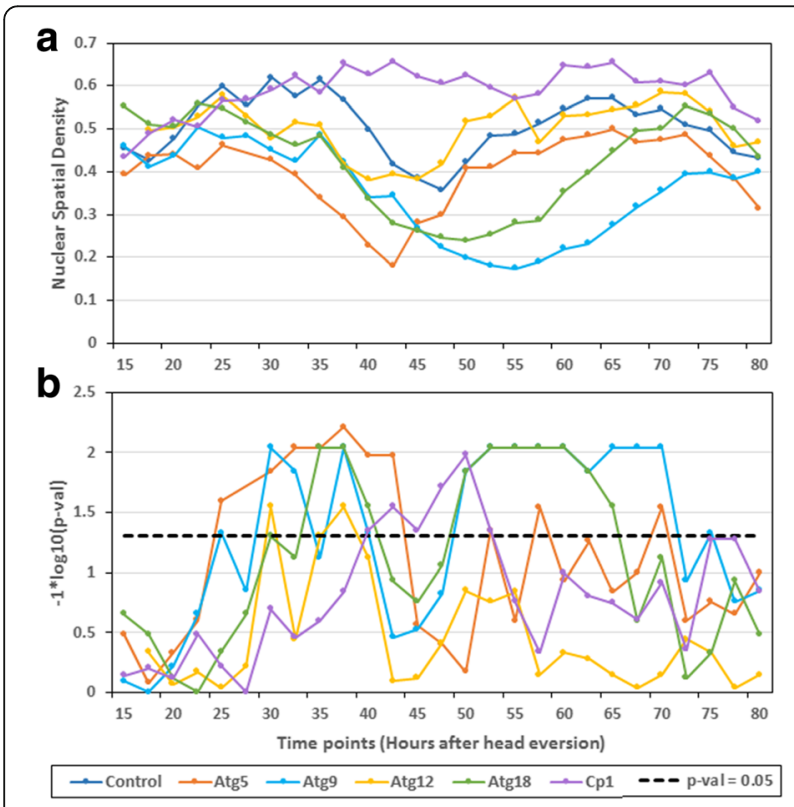

Fig. 15 Nuclear spatial density index quantifies the nuclear distribution with respect to the muscle mass change. a The figure compares average nuclear spatial density index of nuclei in muscles expressing Cp1 RNAi, Atg5 RNAi, Atg9 RNAi, Atg12 RNAi, Atg18 RNAi and control. b Significance graph. Statistics were derived from the same DIOM1 used in previous two figures. The horizontal dotted lines in the significance graphs (bottom pane/s) represent the $p$-value 0.05 processes including nuclear segmentation and tracking are performed in a fully automated fashion, thus enabling a more reproducible analysis of sizeable time-series image data. Nuclear classification results have been improved by introducing a tracking based algorithm which exploits the differences in motion of external and internal nuclei to classify them. Also, the adjustment of position of nuclei based on muscle cell displacement reduced the classification errors caused by large movements of the muscle cell. In some cases, abrupt change in shape of muscle does affect the nuclear tracking result. If the shape change is only between two time points, our algorithm handles this case by creating a new track for the affected nuclei. Therefore, the results of classification are not affected. However, if muscle keeps changing shape for larger duration of time, the performance of classification algorithm will decrease. In our dataset, such cases are very few. In the future, further work can be done to improve the methodology of nuclei adjustment based on displacement of muscle cell centroid. In order to reduce the effect of muscle cell shape change in adjustment of nuclei, a shape matching using criteria like chamfer distance could be used to align muscle cells.

Multiple nuclear spatial pattern features have been designed, each catering to a specific type of nuclear distribution. The nuclear spatial density index measures how densely the nuclei are packed; however, they cannot quantify the localization of the nuclei in the muscle cell. To accomplish this, we have designed new features that can quantify the distribution of nuclei along the medial axis and along the width of the muscle cell, termed longitudinal nuclear spread and lateral nuclear spread respectively.

In this study, the abnormal nuclei arrangement in $C p 1$ and Atgs RNAi has been analyzed statistically using nuclear spatial pattern features. According to the myonuclear domain theory, nuclei should be evenly distributed in healthy muscle [36], which is a phenotype we observed in Cp1 mutant; whereas central positioning of nuclei, which has been associated with Central nuclear myopathies, was observed in control and Atgs RNAi during the mid-pupal stage. Knockdown of $\operatorname{Atg} 9$ and $A \operatorname{tg} 18$ RNAi resulted in more densely packed (longitudinally) nuclei as compared to control. This indicates that Atg9 and Atg18 help in the central positioning of nuclei by regulating their anti-polar migration. We also observed that the effect of silencing of genes on nuclear migration is more prominent in Atg 9 and Atg18 as compared to Atg5 and Atg12. Atgs were also involved in positioning of nuclei along the width of muscle. In order to understand how these genes alter the nuclear spatial pattern, further analysis is required. However, it is clear that the metamorphosis in Drosophila provides a good platform to study nuclear migration and localization in muscles. 


\section{Conclusion}

Understanding the association between the dynamics of myonuclear localization and change in muscle mass requires extensive quantitative analysis of a large number time series images collected in vivo. We achieved it by combining time lapse in vivo imaging of Drosophila metamorphosis with a semi-automated nuclear pattern analysis algorithm. We developed new nuclear features to characterize the dynamics of nuclear distribution in time-lapse images of Drosophila metamorphosis. Image quantification improved our understanding of phenotypic abnormalities in nuclear distribution resulting from gene perturbations. Further analysis on larger number of genes is required to understand in depth the molecular mechanisms behind the myonuclear localization patterns. Therefore in vivo imaging and quantitative image analysis of Drosophila metamorphosis promise to provide novel insights into the relationship between muscle wasting and myonuclear positioning.

\section{Additional file}

Additional file 1: Sample table of nuclear tracks, results of anti-polar/ polar migration analysis and figures showing myonuclear distribution phenotypes observed in previous study. (DOCX 5608 kb)

\begin{abstract}
Abbreviations
AHE: After head eversion; DIOM: Dorsal internal oblique muscle; FMAj: Fly muscle analysis in java; GFP: Green fluorescent protein; LoG: Laplacian of Gaussian; MHC: Myosin heavy chain; MIP: Maximum intensity projection; mKO: Monomeric Kusabira Orange; MND: Myonuclear domain; NSD: Nuclear spatial density; RNAi: RNA interference; TLM: Time-lapse microscopy
\end{abstract}

\section{Acknowledgements}

We thank the TRiP at Harvard Medical School (NIH/NIGMS R01-GM084947) for providing transgenic RNAi fly stocks used in this study. We thank the Bloomington Drosophila stock center for providing fly stocks. We thank Puah Wee Choo for her involvement in laboratory experiments and microscopy. MW and K were affiliated with the BII until 2013 and 2015, respectively.

\section{Funding}

We thank the Bioinformatics Institute, A*STAR, Singapore, for funding the research and Kuleesha's PhD scholarship. The research grant RG139/14 from Ministry of Education, Singapore supported the academic development of this work.

\section{Availability of data and materials}

The software and datasets are available from $\mathrm{K}$. on request.

\section{Authors' contributions}

$\mathrm{K}$ was involved in designing and implementing nuclear spatial pattern analysis pipeline and drafting the manuscript. LF was involved in designing nuclear spatial pattern analysis pipeline and drafting the manuscript. MW conceived and designed the study, was involved in designing and implementing nuclear spatial pattern analysis pipeline, laboratory experiments, microscopy and drafting the manuscript. All authors read and approved the final manuscript

\section{Ethics approval}

Singapore does not require ethics approval for Drosophila research.

\section{Consent for publication}

Not Applicable.

\section{Competing interests}

The authors declare that they have no competing interests.

\section{Publisher's Note}

Springer Nature remains neutral with regard to jurisdictional claims in published maps and institutional affiliations.

\section{Author details}

${ }^{1}$ School of Computer Science and Engineering, Nanyang Technological University, Singapore, Singapore. ${ }^{2}$ Imaging Informatics Division, Bioinformatics Institute (BII), Agency for Science, Technology and Research (A*STAR), Singapore, Singapore. ${ }^{3}$ BiolmagingMW, Block 28D Dover Crescent \#31-73, Singapore 134028, Singapore.

Received: 7 November 2016 Accepted: 22 June 2017

Published online: 10 July 2017

\section{References}

1. Gregory TR. Coincidence, coevolution, or causation? DNA content, cell size, and the C-value enigma. Biol Rev Camb Philos Soc. 2001;76(1):65-101.

2. Gundersen K, Bruusgaard JC. Nuclear domains during muscle atrophy: nuclei lost or paradigm lost? J Physiol. 2008:586(11):2675-81.

3. Priester C, Morton LC, Kinsey ST, Watanabe WO, Dillaman RM. Growth patterns and nuclear distribution in white muscle fibers from black sea bass, Centropristis striata: evidence for the influence of diffusion. J Exp Biol. 2011;214(8):1230-9.

4. Bruusgaard JC, Liestøl K, Ekmark M, Kollstad K, Gundersen K. Number and spatial distribution of nuclei in the muscle fibres of normal mice studied in vivo. J Physiol. 2003;551(2):467-78.

5. Allen DL, Roy RR, Edgerton VR. Myonuclear domains in muscle adaptation and disease. Muscle Nerve. 1999:22(10):1350-60.

6. Wada Kl, Katsuta S, Soya H. Natural occurrence of myofiber cytoplasmic enlargement accompanied by decrease in myonuclear number. Jpn J Physiol. 2003;53(2):145-50.


myopathies. Front Aging Neurosci. 2014;6:339.

8. Gueneau L, et al. Mutations of the FHL1 Gene cause Emery-Dreifuss muscular dystrophy. Am J Hum Genet. 2009;85(3):338-53.

9. $\mathrm{Li} \mathrm{H-H}$, et al. Adeno-associated virus vector carrying human minidystrophin gene SMCKA3999 effectively ameliorates dystrophic pathology in mdx model mice. Zhonghua Yi Xue Za Zhi. 2003;83(17):1513-6.

10. Zhang $X$, et al. Syne-1 and Syne-2 play crucial roles in myonuclear anchorage and motor neuron innervation. Dev Camb Engl. 2007;134(5):901-8.

11. Starr DA, Fridolfsson HN. Interactions between nuclei and the cytoskeleton are mediated by SUN-KASH nuclear-envelope bridges. Annu Rev Cell Dev Biol. 2010:26:421-44.

12. Elhanany-Tamir H, Yu YV, Shnayder M, Jain A, Welte M, Volk T. Organelle positioning in muscles requires cooperation between two KASH proteins and microtubules. J Cell Biol. 2012;198(5):833-46.

13. Schulman VK, Folker ES, Rosen JN, Baylies MK. Syd/JIP3 and JNK signaling are required for Myonuclear positioning and muscle function. PLOS Genet. 2014;10(12):e1004880.

14. Kuleesha Y, Puah WC, Wasser M. A model of muscle atrophy based on live microscopy of muscle remodelling in Drosophila metamorphosis. R Soc Open Sci. 2016;3(2):150517.

15. Cadot B, Gache V, Vasyutina E, Falcone S, Birchmeier C, Gomes ER. Nuclear movement during myotube formation is microtubule and dynein dependent and is regulated by Cdc42, Par6 and Par3. EMBO Rep. 2012;13(8):741-9.

16. Folker ES, Schulman VK, Baylies MK. Translocating myonuclei have distinct leading and lagging edges that require kinesin and dynein. Development 2014;141(2):355-66.

17. Andrey $P$, et al. Statistical analysis of $3 D$ images detects regular spatial distributions of centromeres and chromocenters in animal and plant nuclei. PLoS Comput Biol. 2010;6(7):e1000853.

18. He F, Ma J. A spatial point pattern analysis in Drosophila Blastoderm embryos evaluating the potential inheritance of transcriptional states. PLoS One. 2013;8(4):e60876.

19. Chen $\mathrm{EH}$, Olson EN. Antisocial, an intracellular adaptor protein, is required for Myoblast fusion in Drosophila. Dev Cell. 2001;1(5):705-15. 
20. Karasawa S, Araki T, Nagai T, Mizuno H, Miyawaki A. Cyan-emitting and orange-emitting fluorescent proteins as a donor/acceptor pair for fluorescence resonance energy transfer. Biochem J. 2004;381(1):307.

21. Schnorrer $F$, et al. Systematic genetic analysis of muscle morphogenesis and function in Drosophila. Nature. 2010;464(7286):287-91.

22. Ni J-Q, et al. Vector and parameters for targeted transgenic RNA interference in Drosophila melanogaster. Nat Methods. 2008:5(1):49-51.

23. Kuleesha Y, Puah WC, Lin F, Wasser M. FMAj: a tool for high content analysis of muscle dynamics in Drosophila metamorphosis. BMC Bioinformatics. 2014;15(Suppl 16):S6.

24. Chinta R, Tan JH, Wasser M. The study of muscle remodeling in Drosophila metamorphosis using in vivo microscopy and bioimage informatics. BMC Bioinformatics. 2012;13(Suppl 17):S14.

25. Puah WC, Cheok LP, Biro M, Ng WT, Wasser M. TLM-converter: reorganization of long time-lapse microscopy datasets for downstream image analysis. BioTechniques. 2011;51(1):49-50.

26. Thevenaz P, Sage D, Unser M. Bi-exponential edge-preserving smoother. IEEE Trans Image Process. 2012;21(9):3924-36.

27. "Mexican Hat Filter." [Online]. Available: http://rsb.info.nih.gov/ij/plugins/ mexican-hat/index.html. [Accessed 04 Oct 2015].

28. Al-Kofahi Y, Lassoued W, Lee W, Roysam B. Improved automatic detection and segmentation of cell nuclei in histopathology images. IEEE Trans Biomed Eng. 2010;57(4):841-52.

29. Irshad H, Veillard A, Roux L, Racoceanu D. Methods for nuclei detection, segmentation, and classification in digital histopathology: a review\&amp; \#x2014;current status and future potential. IEEE Rev Biomed Eng. 2014;7:97-114.

30. Chen L, Chan L, Zhao Z, Yan H. A novel cell nuclei segmentation method for 3D C. elegans embryonic time-lapse images. BMC Bioinformatics. 2013;14(1):328.

31. Chen X, Zhou X, Wong STC. Automated segmentation, classification, and tracking of cancer cell nuclei in time-lapse microscopy. IEEE Trans Biomed Eng. 2006;53(4):762-6.

32. Bao Z, Murray Jl, Boyle T, Ooi SL, Sandel MJ, Waterston RH. Automated cell lineage tracing in Caenorhabditis elegans. Proc Natl Acad Sci. 2006; 103(8):2707-12

33. Wagner T, Lipinski H-G. IJBlob: An ImageJ Library for Connected Component Analysis and Shape Analysis. J Open Res Softw. (2013);1(1):e6. doi: http://doi.org/10.5334/jors.ae

34. Schindelin J, et al. Fiji: an open-source platform for biological-image analysis. Nat Methods. 2012;9(7):676-82.

35. "Hull And Circle." [Online]. Available: http://rsb.info.nih.gov/ij/plugins/hullcircle.html. [Accessed 03 Oct 2015].

36. Hall ZW, Ralston E. Nuclear domains in muscle cells. Cell. 1989;59(5):771-2.

\section{Submit your next manuscript to BioMed Central and we will help you at every step:}

- We accept pre-submission inquiries

- Our selector tool helps you to find the most relevant journal

- We provide round the clock customer support

- Convenient online submission

- Thorough peer review

- Inclusion in PubMed and all major indexing services

- Maximum visibility for your research

Submit your manuscript at www.biomedcentral.com/submit 